This item is the archived peer-reviewed author-version of:

\title{
Uniform strain in heterostructure tunnel field-effect transistors
}

\section{Reference:}

Verreck Devin, Verhulst Anne S., Van de Put Maarten, Sorée Bart, Collaert Nadine, Mocuta Anda, Thean Aaron, Groeseneken Guido.- Uniform strain in heterostructure tunnel field-effect transistors

IEEE electron device letters - ISSN 0741-3106 - 37:3(2016), p. 337-340

Full text (Publishers DOI): http://dx.doi.org/doi:10.1109/LED.2016.2519681

To cite this reference: http://hdl.handle.net/10067/1332070151162165141 


\title{
Uniform strain in heterostructure tunnel field-effect transistors
}

\author{
Devin Verreck, Anne S. Verhulst, Maarten L. Van de Put, Bart Sorée, Nadine Collaert, Anda Mocuta, \\ Aaron Thean, and Guido Groeseneken
}

\begin{abstract}
Strain can strongly impact the performance of III-V tunnel field-effect transistors (TFET). However, previous studies on homostructure TFETs have found an increase in on-current to be accompanied with a degradation of subthreshold swing. We perform 30-band quantum mechanical simulations of staggered heterostructure p-n-i-n tunnel field-effect transistors submitted to uniaxial and biaxial uniform stress and find the origin of the subthreshold degradation to be a reduction of the density of states in the strained case. We apply an alternative configuration including a lowly doped pocket in the source which allows to take full benefit of the strain-induced increase in on-current.
\end{abstract}

Index Terms-TFET, strain, heterostructure

B AND-TO-BAND tunneling (BTBT) allows the tunnel field-effect transistor (TFET) to obtain a sub- $60 \mathrm{mV} / \mathrm{dec}$ subthreshold swing (SS) at room temperature [1]-[3]. This enables a reduction of supply voltage, making the TFET a promising alternative to the conventional metal-oxidesemiconductor field-effect transistor (MOSFET) for future low-power applications. The challenge consists, however, in sustaining the sub-60 $\mathrm{mV} / \mathrm{dec} \mathrm{SS}$ up to sufficiently high currents. Recent research has therefore proposed various improvements to the classic Si p-i-n TFET design in the form of dopant pockets [4], [5], alternative gate positioning and composition [6]-[8], a change in material to III-V compounds and the introduction of a heterostructure [9]-[14].

Strain engineering is another way to influence TFET performance through its effect on the band structure. In current MOSFET technology, the application of strain has become an invaluable asset to increase carrier mobilities [15]. For TFET, the effect of strain on the tunneling current has been simulated [16], [17] and measured [18]-[21] in group-IV configurations, with biaxial tensile strain turning out beneficial for the oncurrent $\left(\mathrm{I}_{\mathrm{ON}}\right)$ and SS. For III-V TFETs, strain research is more scarce. 8-band k.p simulation studies have predicted a beneficial effect on $\mathrm{I}_{\mathrm{ON}}$ for biaxial tensile strain, both in InAs homostructure [22] and broken-gap GaSb/InAs heterostructure TFETs [23]. However, in both cases the SS was found to deteriorate with increasing tensile strain.

D.Verreck acknowledges support from the Agency for Innovation by Science and Technology in Flanders (IWT). This work was supported by imec's Industrial Affiliation Program.

D.Verreck and G.Groeseneken are with imec, 3001 Leuven, Belgium and also with the Department of Electrical Engineering, KU Leuven, 3001 Leuven, Belgium (e-mail: devin.verreck@imec.be).

A.S.Verhulst, A.Mocuta, N.Collaert and A.Thean are with imec, 3001 Leuven, Belgium.

M.L.Van de Put and Bart Sorée are with imec, 3001 Leuven, Belgium and also with the Department of Physics, Universiteit Antwerpen, 2020 Antwerpen, Belgium.
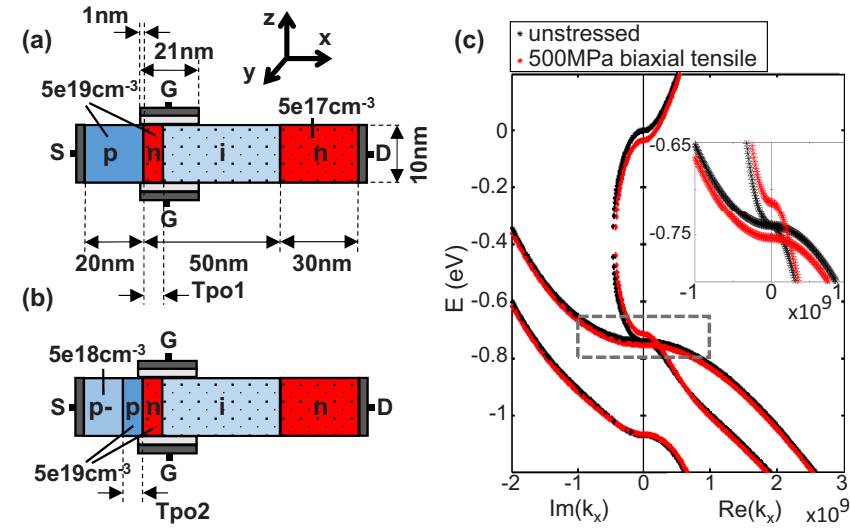

Fig. 1. Simulated TFET configurations: (a) p-n-i-n, (b) $\mathrm{p}^{-} \mathrm{p}^{+}-\mathrm{n}-\mathrm{i}-\mathrm{n}$, both with an effective oxide thickness of $0.6 \mathrm{~nm}$ and a gate workfunction of $5 \mathrm{eV}$. The solid and dotted regions consist of respectively $\mathrm{GaAs}_{0.5} \mathrm{Sb}_{0.5}$ and $\mathrm{In}_{0.53} \mathrm{Ga}_{0.47}$ As. The y-direction is translationally invariant. (c) unstrained and strained band structure of $\operatorname{In}_{0.53} \mathrm{Ga}_{0.47}$ As. Inset: enlargement of the boxed region.

In this letter, we investigate the cause of the SS degradation by means of quantum mechanical (QM) 30-band k.pbased simulations of staggered $\mathrm{GaAs}_{0.5} \mathrm{Sb}_{0.5} / \mathrm{In}_{0.53} \mathrm{Ga}_{0.47} \mathrm{As}$ heterostructure TFETs and suggest an alternative configuration to counteract the SS degradation while improving $\mathrm{I}_{\mathrm{ON}}$.

We use a 2D ballistic QM solver, based on the 30-band envelope function formalism [24], [25]. The solver uses a wavefunction approach and assumes transmitting boundary conditions at the source and drain contacts. Compared to an 8-band model, the 30-band model can capture the full 1st Brillouin zone and does not require perturbative Luttinger parameters. The $\mathbf{k} \cdot \mathbf{p}$-parameters used in this work are given in Tables I and II. The coupling parameter $\mathrm{E}_{\mathrm{P}}$ for $\mathrm{In}_{0.53} \mathrm{Ga}_{0.47} \mathrm{As}$ is calibrated to experimental diode results [26]. The other k.pparameters are fitted to bandgaps and effective masses for the $\Gamma$-valley and bandgaps for the $\mathrm{X}$ - and L-valleys [27], while retaining commutativity of the momentum matrices [28]. To account for the difference in basis functions in the different materials of the heterostructure, the momentum and Hamiltonian matrix elements are transformed [29].

The effects of strain on the band structure (see Fig. 1), are captured through the strain Hamiltonian $H_{S}$, given in the Appendix. We consider only uniform normal strain, which means the strain tensor is diagonal and invariant.

The electrostatic potential is obtained from a semi-classical (SC) solution of the Poisson and carrier continuity equations [30]. To incorporate the effect of strain on the potential, we 
TABLE I

30-BAND K·P BASIS FUNCTIONS AND ENERGY LEVELS. NOTATION FROM RADHIA [31].

\begin{tabular}{|c|c|c|c|}
\hline $\begin{array}{l}\text { Basis } \\
\text { functions }\end{array}$ & $\begin{array}{l}\text { Energy } \\
\text { levels }\end{array}$ & $\begin{array}{c}\mathrm{In}_{0.53} \mathrm{Ga}_{0.47} \mathrm{As} \\
{[\mathrm{eV}]}\end{array}$ & $\begin{array}{c}\mathrm{GaAs}_{0.5} \mathrm{Sb}_{0.5} \\
{[\mathrm{eV}]}\end{array}$ \\
\hline$\left|S_{V}\right\rangle$ & $\Gamma_{6 V}$ & -12.55 & -12.17 \\
\hline & $\Gamma_{7 V}$ & -0.33 & -0.4 \\
\hline$|X\rangle,|Y\rangle,|Z\rangle$ & $\Gamma_{8 V}$ & 0 & 0 \\
\hline$|S\rangle$ & $\Gamma_{6 C}$ & 0.74 & 0.72 \\
\hline$\left.\left.\left|X_{\infty}\right\rangle, Y_{c}\right\rangle, Z_{\beta}\right\rangle$ & $\Gamma_{7 C}$ & 4.33 & 3.89 \\
\hline$\left|X_{c}\right\rangle,\left|Y_{c}\right\rangle,\left|Z_{c}\right\rangle$ & $\Gamma_{8 C}$ & 4.33 & 4.07 \\
\hline$\left|S_{u}\right\rangle$ & $\Gamma_{6 u}$ & 8.55 & 8.56 \\
\hline$\left|D_{z}\right\rangle,\left|D_{r}\right\rangle$ & $\Gamma_{8-3}$ & 10.02 & 9.59 \\
\hline & $\Gamma_{8-3}$ & 10.02 & 10.17 \\
\hline$\left|X_{d}\right\rangle,\left|Y_{d}\right\rangle,\left|Z_{d}\right\rangle$ & $\Gamma_{7 d}$ & 11.89 & 10.99 \\
\hline$\left|S_{q}\right\rangle$ & $\begin{array}{l}\Gamma_{8 d} \\
\Gamma_{6 q}\end{array}$ & $\begin{array}{l}11.89 \\
13.11\end{array}$ & $\begin{array}{l}10.99 \\
13.19\end{array}$ \\
\hline
\end{tabular}

TABLE II

30-BAND K·P MOMENTUM MATRIX ELEMENTS FOR IN $\mathrm{IN}_{0.53} \mathrm{GA}_{0.47} \mathrm{AS}$ (I) AND GAAs G.5 $_{0.5} \mathrm{SB}_{0.5}$ (II). NOTATION FROM RADHIA [31].

\begin{tabular}{|l||c|c||l||c|c|}
\hline Element & $\mathrm{I}[\mathrm{eV}]$ & $\mathrm{II}[\mathrm{eV}]$ & Element & $\mathrm{I}[\mathrm{eV}]$ & $\mathrm{II}[\mathrm{eV}]$ \\
\hline $\mathrm{E}_{P}$ & 19.33 & 17.09 & $\mathrm{E}_{P d}$ & 0.06 & 1.14 \\
$\mathrm{E}_{P X}$ & 13.96 & 13.01 & $\mathrm{E}_{P X d}$ & 5.71 & 3.76 \\
$\mathrm{E}_{P 3}$ & 3.22 & 3.00 & $\mathrm{E}_{P 3 d}$ & 7.86 & 10.41 \\
$\mathrm{E}_{P 2}$ & 0 & 0 & $\mathrm{E}_{P 2 d}$ & 21.37 & 24.32 \\
$\mathrm{E}_{P S}$ & 0.05 & 0.12 & $\mathrm{E}_{P U}$ & 19.62 & 16.20 \\
$\mathrm{E}_{P^{\prime}}$ & 0.01 & $1.15 \times 10^{-4}$ & & & \\
\hline
\end{tabular}

have inserted the effective masses from a strained 30-band $\mathbf{k} \cdot \mathbf{p}$ model into the SC simulator, with a non-parabolic correction.

The simulated configurations are double gate $\mathrm{GaAs}_{0.5} \mathrm{Sb}_{0.5} / \mathrm{In}_{0.53} \mathrm{Ga}_{0.47} \mathrm{As}$ p-n-i-n TFETs, displayed in Fig. 1. These configurations can be grown vertically with molecular beam epitaxy [4]. The channel length has been chosen sufficiently long to suppress source-drain tunneling, while the body thickness is sufficiently wide to reduce the effect of size-induced quantization. All doping profiles are abrupt: dopant fluctuation and variability are not taken into account. In a p-n-i-n TFET, a counterdoped pocket is added to increase the electric field at the tunnel junction [5]. This also reduces the impact of field-induced quantization, which might influence the device electrostatics, as it forces tunnel paths more parallel to the gate. The staggered heterojunction provides an additional performance improvement thanks to the small effective bandgap at the tunnel junction (see Fig. 2).

We now apply $500 \mathrm{MPa}$ of compressive and tensile uniaxial and tensile biaxial stress to the $3 \mathrm{~nm}$ and $0 \mathrm{~nm}$ pocket configu-

TABLE III

ENERGY GAPS AND EFFECTIVE MASSES IN BULK FOR ELECTRONS(EL), LIGHT HOLES(LH) AND HEAVY HOLES(HH) AT THE $\Gamma$-POINT, UNSTRAINED AND UNDER BIAXIAL TENSILE STRESS.

\begin{tabular}{|l||c|c|c|c|c|c|}
\hline \multicolumn{1}{|c||}{} & \multicolumn{2}{c|}{$\mathrm{In}_{0.53} \mathrm{Ga}_{0.47} \mathrm{As}$} & \multicolumn{3}{c|}{$\mathrm{GaAs}_{0.5} \mathrm{Sb}_{0.5}$} \\
\hline$[\mathrm{eV}]$ & $0 \mathrm{MPa}$ & \multicolumn{2}{|c|}{$500 \mathrm{MPa}$} & $0 \mathrm{MPa}$ & \multicolumn{2}{c|}{$500 \mathrm{MPa}$} \\
\hline $\mathrm{E}_{g}$ & 0.74 & \multicolumn{2}{|c|}{0.68} & 0.72 & \multicolumn{2}{c|}{0.68} \\
$\Delta_{l h-h h}$ & 0 & \multicolumn{2}{c|}{0.04} & 0 & \multicolumn{2}{c|}{0.04} \\
$\Delta_{s o}$ & 0.33 & \multicolumn{2}{c|}{0.35} & 0.40 & \multicolumn{2}{c|}{0.42} \\
\hline$\left[\mathrm{m}_{0}\right]$ & $x, y, z$ & $x$ & $y, z$ & $x, y, z$ & $x$ & $y, z$ \\
\hline$m_{e l}^{*}$ & 0.041 & 0.037 & 0.040 & 0.046 & 0.041 & 0.040 \\
$m_{l h}^{*}$ & 0.055 & 0.046 & 0.174 & 0.060 & 0.051 & 0.181 \\
$m_{h h}^{*}$ & 0.450 & 0.452 & 0.068 & 0.455 & 0.458 & 0.074 \\
$m_{s o}^{*}$ & 0.135 & 0.154 & 0.121 & 0.155 & 0.172 & 0.142 \\
\hline
\end{tabular}

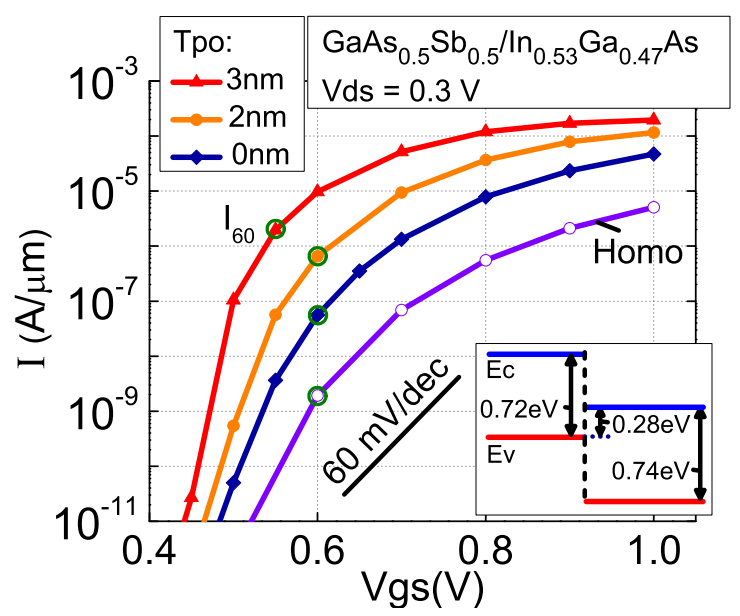

Fig. 2. QM I-V curves of p-n-i-n hetero-TFETs with varying Tpo1. For comparison, an $\operatorname{In}_{0.53} \mathrm{Ga}_{0.47} \mathrm{As} \mathrm{p}$-i-n homo-TFET is also included. Inset: band alignment at the source-channel junction. Configuration details in Fig. 1.

rations of Fig. 2 (uniaxial: $\sigma_{x x}= \pm 500 \mathrm{MPa}, \sigma_{y y}=\sigma_{z z}=0$, biaxial: $\sigma_{x x}=0, \sigma_{y y}=\sigma_{z z}=500 \mathrm{MPa}$ ). The uniaxial stress could be realized in suspended nanowires [32], [33], while the biaxial tensile stress could be the result of lattice mismatch with an underlying substrate. Fig. 3 shows the main effect is a deterioration in SS, although for the biaxial case somewhat higher current densities are reached for high $\mathrm{V}_{\mathrm{gs}}$ than for the other cases. The band diagrams in Fig. 4(a) illustrate that the SS degradation is caused by an increase in source degeneracy for all strain configurations, which leaves the exponential tail of the Fermi-Dirac distribution partially uncovered. The larger degeneracy results from a decrease in density of states (DOS). A comparison of effective masses and energy gaps at the $\Gamma$ point between the unstrained and the biaxial tensile case is presented in Table III, showing a sharp decrease especially for the heavy hole mass in the $y$-and $z$-directions. Additionally, the band structure in Fig. 1 shows that the strain lifts the degeneracy between the heavy and light hole bands, further decreasing the DOS. Note that the strained imaginary branches in Fig. 1 show that only the light hole band couples with the first conduction band, thereby determining the effective tunneling bandgap. The higher current densities at high $\mathrm{V}_{\mathrm{gs}}$ are a consequence of a decrease in this bandgap compared to the unstrained case. Although this current increase could be beneficial for $\mathrm{I}_{\mathrm{ON}}$, it is clear that the SS degradation severely limits the utility of stress in improving TFET performance.

The increase in source doping degeneracy due to strain can be counteracted by the introduction of a lowly doped source region, while keeping the doping high at the tunnel junction, creating a $\mathrm{p}^{-} \mathrm{p}^{+}-\mathrm{n}-\mathrm{i}-\mathrm{n}$ structure (see Fig. 1(b)). This concept has been previously suggested as a solution for the poor performance of p-type III-V TFETs [24]. If we apply it to strained n-TFETs, we can benefit from the reduction in source doping degeneracy (see Fig. 4(b)) to avoid the SS degradation, while maintaining improvements in $\mathrm{I}_{\mathrm{ON}}$. Fig. 5 shows that with the improved source design, an $\mathrm{I}_{60}$, the current at which the SS goes from sub-to super- $60 \mathrm{mV} / \mathrm{dec}$, of $10 \mu \mathrm{A} / \mu \mathrm{m}$ with an $\mathrm{I}_{\mathrm{ON}}$ of $140 \mu \mathrm{A} / \mu \mathrm{m}$ can be obtained with $500 \mathrm{MPa}$ of 


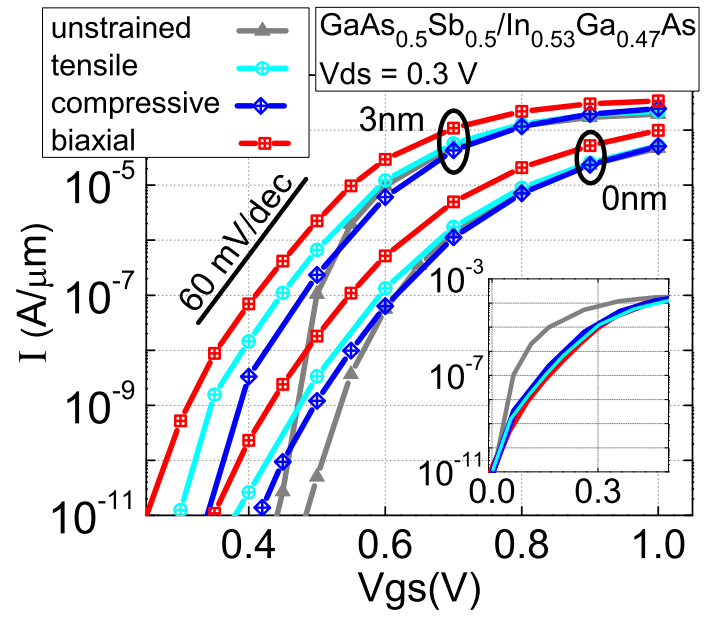

Fig. 3. QM I-V curves of stressed p-n-i-n hetero-TFETs for a Tpo1 of $3 \mathrm{~nm}$ and $0 \mathrm{~nm}$. Inset: $3 \mathrm{~nm}$ curves shifted to the same $\mathrm{I}_{\mathrm{OFF}}$. Configuration details in Fig. 1.
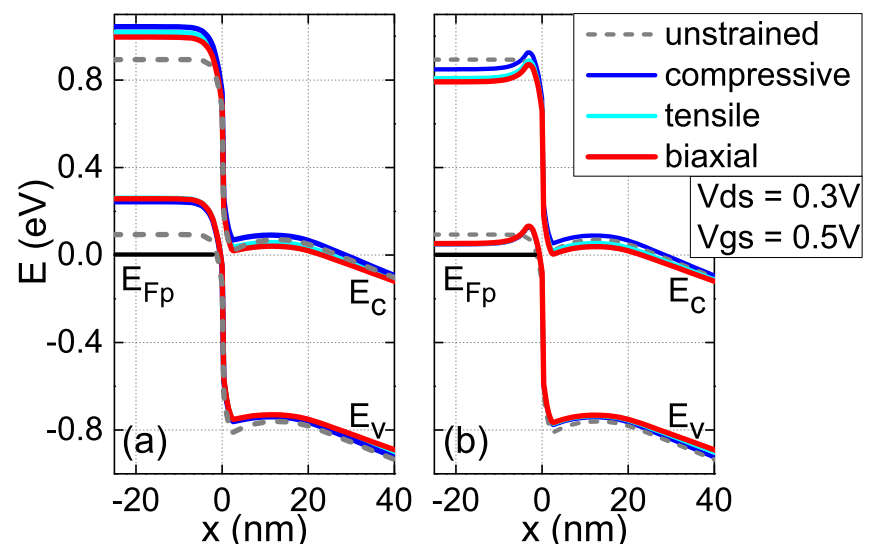

Fig. 4. Band diagrams of the first subband around the tunnel junction for different types of strain along a cutline through the body center of (a) a p-ni-n TFET with a Tpol of $3 \mathrm{~nm}$ and (b) a $\mathrm{p}^{-} \mathrm{p}^{+}-\mathrm{n}$-i-n TFET with a Tpo1 of $3 \mathrm{~nm}$ and a Tpo 2 of $4 \mathrm{~nm}$. The black line shows the hole Fermi level $\left(\mathrm{E}_{\mathrm{Fp}}\right)$.

biaxial tensile stress. With $\mathrm{I}_{\mathrm{OFF}}$ at $10 \mathrm{pA} / \mu \mathrm{m}, \mathrm{I}_{\mathrm{ON}}$ is defined at $\mathrm{V}_{D D}=0.3 \mathrm{~V}$ above the onset voltage. Increasing the stress to $1 \mathrm{GPa}$ improves $\mathrm{I}_{\mathrm{ON}}$ further to $170 \mu \mathrm{A} / \mu \mathrm{m}$, with a similar $\mathrm{I}_{60}$ as for the $500 \mathrm{MPa}$ case. An identical unstrained $\mathrm{p}^{-} \mathrm{p}^{+}$$\mathrm{n}$-i-n structure has an $\mathrm{I}_{\mathrm{ON}}$ of $110 \mu \mathrm{A} / \mu \mathrm{m}$ (not shown), with the shifted I-V coinciding up to $30 \mu \mathrm{A} / \mu \mathrm{m}$, since the SS is determined by the source degeneracy as the tunnel paths are only a couple of nm (see Fig. 4). The I I improvement in the strained case is limited, but in such a nearly optimal design this is to be expected. Nevertheless, our results show that we can use the source concept to benefit fully from the straininduced $\mathrm{I}_{\mathrm{ON}}$ improvement, while avoiding a degradation in SS. It also confirms the origin of the SS degradation to be the reduction of the DOS in the source region, rather than a change in band structure. For p-TFET, the reduction in DOS is much smaller, as it is determined by the conduction band in the source. We have found that an optimized unstrained $\mathrm{n}^{-} \mathrm{n}^{+}$-p-i-p TFET therefore directly benefits from strain.

In conclusion, we have shown that strain is indeed a promising performance booster for TFET, using 30-band

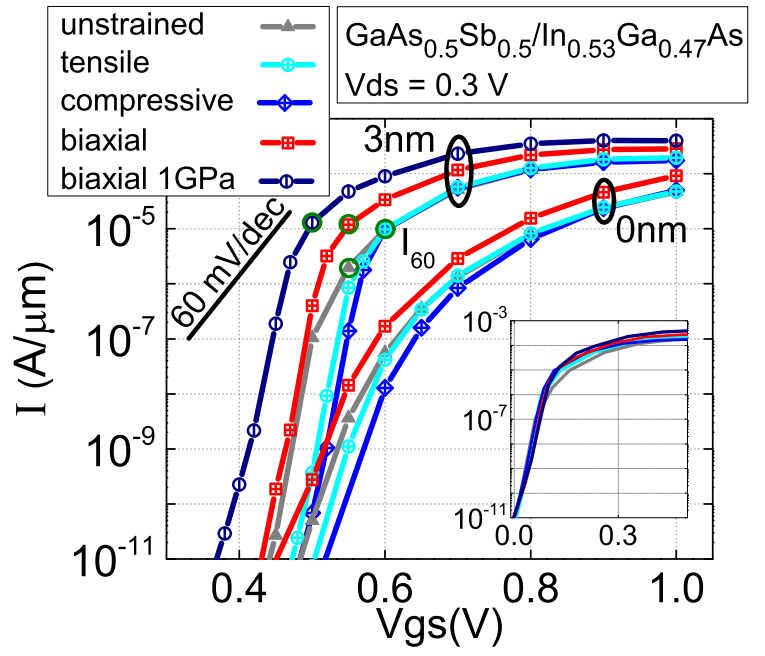

Fig. 5. QM I-V curves of an unstressed p-n-i-n TFET and stressed $p^{-} p^{+}-n-$ i-n hetero-TFETs for a Tpo1 of $3 \mathrm{~nm}$ and $0 \mathrm{~nm}$. Tpo2 is always $4 \mathrm{~nm}$. Inset: $3 \mathrm{~nm}$ curves shifted to the same $\mathrm{I}_{\mathrm{OFF}}$. Configuration details in Fig. 1 .

QM simulations of strained staggered heterostructure p-ni-n TFETs. The degradation in SS which plagues TFETs under uniaxial or biaxial stress is a result of a strain-induced reduction in DOS and can be counteracted with an improved source design. Introducing a lowly doped source region, while maintaining the high doping at the tunnel junction reduces the source degeneracy and allows to take full benefit of the $\mathrm{I}_{\mathrm{ON}}$ improvement under biaxial tensile stress.

Our work shows that stress can be an alternative to an increase in maximal source doping level. Future research should focus on integrating these findings into a full analysis of non-uniform strain conditions.

\section{APPENDIX A}

The 8-band k·p strain Hamiltonian matrix by Bahder [34] was used by Neffati et al. for full-zone models at small kvalues [35]. We consider a uniform strain tensor:

$$
\overline{\bar{\varepsilon}}=\left[\begin{array}{ccc}
\varepsilon_{\perp} & 0 & 0 \\
0 & \varepsilon_{\|} & 0 \\
0 & 0 & \varepsilon_{\|}
\end{array}\right]
$$

with $\varepsilon_{\perp}$ and $\varepsilon_{\|}$the strain components in respectively the $x$ and $y, z$-directions. In the basis of Table I, the strain Hamiltonian is

$$
\mathbf{H}_{S}=\operatorname{diag}\left[\begin{array}{c}
a_{c} \operatorname{Tr}(\overline{\bar{\varepsilon}}) \\
a_{v} \operatorname{Tr}(\overline{\bar{\varepsilon}})+\mathrm{b}_{\mathrm{v}} \varepsilon_{\| \perp} \\
a_{v} \operatorname{Tr}(\overline{\bar{\varepsilon}})+\mathrm{b}_{\mathrm{v}} \varepsilon_{\| \perp} \\
a_{v} \operatorname{Tr}(\overline{\bar{\varepsilon}})-2 \mathrm{~b}_{\mathrm{v}} \varepsilon_{\| \perp} \\
a_{c} \operatorname{Tr}(\overline{\bar{\varepsilon}}) \\
a_{v} \operatorname{Tr}(\overline{\bar{\varepsilon}})+\mathrm{b}_{\mathrm{v}} \varepsilon_{\| \perp} \\
a_{v} \operatorname{Tr}(\overline{\bar{\varepsilon}})+\mathrm{b}_{\mathrm{v}} \varepsilon_{\| \perp} \\
a_{v} \operatorname{Tr}(\overline{\bar{\varepsilon}})-2 \mathrm{~b}_{\mathrm{v}} \varepsilon_{\| \perp}
\end{array}\right]
$$

where $\varepsilon_{\| \perp}=\varepsilon_{\|}-\varepsilon_{\perp}$ and $a_{c}, a_{v}$ and $b_{v}$ are deformation potentials, obtained from Vurgaftman et al. [36]. $a_{c}$ and $b_{v}$ are positive and $a_{v}$ is negative, such that a positive (negative) strain is compressive (tensile). To calculate $\overline{\bar{\varepsilon}}$ from the applied stress, we use stiffness coefficients from Goldberg et al. [27]. 


\section{REFERENCES}

[1] H. Lu and A. Seabaugh, "Tunnel field-effect transistors: State-of-theart," IEEE J. Electron Dev. Soc., vol. 2, no. 4, pp. 44-49, July 2014. doi: $10.1109 / \mathrm{JEDS} .2014 .2326622$

[2] A. M. Ionescu and H. Riel, "Tunnel field-effect transistors as energyefficient electronic switches." Nature, vol. 479, no. 7373, pp. 329-37, Nov. 2011. doi: 10.1038/nature10679

[3] U. Avci, D. Morris, and I. Young, "Tunnel field-effect transistors: Prospects and challenges," Electron Devices Society, IEEE Journal of the, vol. 3, no. 3, pp. 88-95, May 2015. doi: 10.1109/JEDS.2015.2390591

[4] A. Tura, Z. Zhang, P. Liu, Y.-H. Xie, and J. Woo, "Vertical silicon pn-p-n tunnel nMOSFET with MBE-grown tunneling junction," Electron Devices, IEEE Transactions on, vol. 58, no. 7, pp. 1907-1913, July 2011. doi: 10.1109/TED.2011.2148118

[5] D. Verreck, A. Verhulst, K.-H. Kao, W. Vandenberghe, K. De Meyer, and G. Groeseneken, "Quantum mechanical performance predictions of p-n-i-n versus pocketed line tunnel field-effect transistors," IEEE Trans. Electron Dev., vol. 60, no. 7, pp. 2128-2134, Oct. 2013. doi: 10.1109/TED.2013.2260237

[6] A. S. Verhulst, W. G. Vandenberghe, K. Maex, and G. Groeseneken, "Tunnel field-effect transistor without gate-drain overlap," Applied Physics Letters, vol. 91, no. 5, p. 053102, July 2007. doi: $10.1063 / 1.2757593$

[7] K. Ganapathi and S. Salahuddin, "Heterojunction vertical band-to-band tunneling transistors for steep subthreshold swing and high on current," Electron Device Letters, IEEE, vol. 32, no. 5, pp. 689-691, May 2011. doi: 10.1109/LED.2011.2112753

[8] S. Saurabh and M. Kumar, "Novel attributes of a dual material gate nanoscale tunnel field-effect transistor," Electron Devices, IEEE Transactions on, vol. 58, no. 2, pp. 404-410, Feb. 2011. doi: 10.1109/TED.2010.2093142

[9] S. Koswatta, "On the possibility of obtaining MOSFET-like performance and sub-60-mV/dec swing in 1-D broken-gap tunnel transistors," IEEE Trans. Electron Dev., vol. 57, no. 12, pp. 3222-3230, Dec. 2010. doi: 10.1109/TED.2010.2079250

[10] G. Dewey, B. Chu-Kung, J. Boardman, J. M. Fastenau, J. Kavalieros, R. Kotlyar, W. K. Liu, D. Lubyshev, M. Metz, N. Mukherjee, P. Oakey, R. Pillarisetty, M. Radosavljevic, H. W. Then, and R. Chau, "Fabrication, characterization, and physics of IIIV heterojunction tunneling field-effect transistors (H-TFET) for steep sub-threshold swing," Int. Electron Dev. Meet., vol. 3, pp. 33.6.1-33.6.4, Dec. 2011. doi: 10.1109/IEDM.2011.6131666

[11] K. Tomioka and T. Fukui, "Current increment of tunnel field-effect transistor using InGaAs nanowire/Si heterojunction by scaling of channel length," Appl. Phys. Lett., vol. 104, no. 7, p. 073507, Feb. 2014. doi: $10.1063 / 1.4865921$

[12] J. Knoch and J. Appenzeller, "Modeling of high-performance p-type IIIV heterojunction tunnel FETs," IEEE Electron Dev. Lett., vol. 31, no. 4, pp. 305-307, Apr. 2010. doi: 10.1109/LED.2010.2041180

[13] E. Lind, E. Memisevic, A. Dey, and L.-E. Wernersson, "III-V heterostructure nanowire tunnel FETs," Electron Devices Society, IEEE Journal of the, vol. 3, no. 3, pp. 96-102, May 2015. doi: 10.1109/JEDS.2015.2388811

[14] D. Cutaia, K. Moselund, M. Borg, H. Schmid, L. Gignac, C. Breslin, S. Karg, E. Uccelli, and H. Riel, "Vertical InAs-Si gate-all-around tunnel FETs integrated on Si using selective epitaxy in nanotube templates," Electron Devices Society, IEEE Journal of the, vol. 3, no. 3, pp. 176183, May 2015. doi: 10.1109/JEDS.2015.2388793

[15] M. Chu, Y. Sun, U. Aghoram, and S. E. Thompson, "Strain: A Solution for Higher Carrier Mobility in Nanoscale MOSFETs," Annual Review of Materials Research, vol. 39, pp. 203-229, Aug. 2009. doi: 10.1146/annurev-matsci-082908-145312

[16] K.-H. Kao, A. S. Verhulst, M. Van de Put, W. G. Vandenberghe, B. Sorée, W. Magnus, and K. De Meyer, "Tensile strained Ge tunnel field-effect transistors: k.p material modeling and numerical device simulation," Journal of Applied Physics, vol. 115, no. 4, p. 044505 , Jan. 2014. doi: 10.1063/1.4862806

[17] R. Kotlyar, U. E. Avci, S. Cea, R. Rios, T. D. Linton, K. J. Kuhn, and I. A. Young, "Bandgap engineering of group IV materials for complementary $\mathrm{n}$ and $\mathrm{p}$ tunneling field effect transistors," Applied Physics Letters, vol. 102, no. 11, p. 113106, March 2013. doi: 10.1063/1.4798283

[18] P.-F. Guo, L.-T. Yang, Y. Yang, L. Fan, G.-Q. Han, G. Samudra, and Y.-C. Yeo, "Tunneling field-effect transistor: Effect of strain and temperature on tunneling current," Electron Device Letters, IEEE, vol. 30, no. 9, pp. 981-983, Sept. 2009. doi: 10.1109/LED.2009.2026296
[19] P. Solomon, I. Lauer, A. Majumdar, J. Teherani, M. Luisier, J. Cai, and S. Koester, "Effect of uniaxial strain on the drain current of a heterojunction tunneling field-effect transistor," Electron Device Letters, IEEE, vol. 32, no. 4, pp. 464-466, April 2011. doi: 10.1109/LED.2011.2108993

[20] L. Knoll, Q.-T. Zhao, A. Nichau, S. Trellenkamp, S. Richter, A. Schafer, D. Esseni, L. Selmi, K. Bourdelle, and S. Mantl, "Inverters with strained Si nanowire complementary tunnel field-effect transistors," Electron Device Letters, IEEE, vol. 34, no. 6, pp. 813-815, June 2013. doi: 10.1109/LED.2013.2258652

[21] G. Han, Y. Wang, Y. Liu, H. Wang, M. Liu, C. Zhang, J. Zhang, B. Cheng, and Y. Hao, "Relaxed germanium-tin p-channel tunneling field-effect transistors fabricated on Si: impacts of Sn composition and uniaxial tensile strain," AIP Advances, vol. 5, no. 5, p. 057145, May 2015. doi: 10.1063/1.4921572

[22] F. Conzatti, M. G. Pala, D. Esseni, E. Bano, and L. Selmi, "StrainInduced Performance Improvements in InAs Nanowire Tunnel FETs," IEEE Transactions on Electron Devices, vol. 59, no. 8, pp. 2085-2092, Aug. 2012. doi: 10.1109/TED.2012.2200253

[23] S. Brocard, M. G. Pala, and D. Esseni, "Design options for heterojunction tunnel FETs with high on current and steep sub-threshold voltage slope," in 2013 IEEE International Electron Devices Meeting. IEEE, Dec. 2013. doi: 10.1109/IEDM.2013.6724567 pp. 5.4.1-5.4.4.

[24] D. Verreck, M. Van de Put, B. Sorée, A. S. Verhulst, W. Magnus, W. G. Vandenberghe, N. Collaert, A. Thean, and G. Groeseneken, "Quantum mechanical solver for confined heterostructure tunnel fieldeffect transistors," Journal of Applied Physics, vol. 115, no. 5, p. 053706, Feb. 2014. doi: $10.1063 / 1.4864128$

[25] D. Verreck, A. S. Verhulst, M. Van de Put, B. Sorée, W. Magnus, A. Mocuta, N. Collaert, A. Thean, and G. Groeseneken, "Full-zone spectral envelope function formalism for the optimization of line and point tunnel field-effect transistors," Journal of Applied Physics, vol. 118, no. 13, p. 134502 , Oct. 2015. doi: 10.1063/1.4931890

[26] Q. Smets, D. Verreck, A. S. Verhulst, R. Rooyackers, C. Merckling, M. Van De Put, E. Simoen, W. Vandervorst, N. Collaert, V. Y. Thean, B. Sorée, G. Groeseneken, and M. M. Heyns, "InGaAs tunnel diodes for the calibration of semi-classical and quantum mechanical band-to-band tunneling models," J. Appl. Phys., vol. 115, no. 18, p. 184503, May 2014. doi: $10.1063 / 1.4875535$

[27] Y. A. Goldberg, N. M. Shmidt, and Y. A. Vul, Handbook Series of Semiconductor Parameters, 1999, vol. 2, ch. 3,5.

[28] D. Verreck, M. Van de Put, A. S. Verhulst, B. Sorée, W. Magnus, A. Dabral, A. Thean, and G. Groeseneken, "15-band spectral envelope function formalism applied to broken gap tunnel field-effect transistors," Sept. 2015. doi: 10.1109/IWCE.2015.7301988

[29] M. L. Van de Put, W. G. Vandenberghe, W. Magnus, and B. Sorée, "An envelope function formalism for lattice-matched heterostructures," Physica B: Condensed Matter, vol. 470, pp. 69 - 75, Aug. 2015. doi: 10.1016/j.physb.2015.04.031

[30] Synopsys, Sentaurus Device User Guide, 2013.12.

[31] S. B. Radhia, N. Fraj, I. Saidi, and K. Boujdaria, "The eight-level k.p model for the conduction and valence bands of InAs, InP, InSb," Semicond. Sci. Technol., vol. 22, no. 4, p. 427, Apr. 2007. doi: 10.1088/0268$1242 / 22 / 4 / 024$

[32] R. A. Minamisawa, M. J. Süess, R. Spolenak, J. Faist, C. David, J. Gobrecht, K. K. Bourdelle, and H. Sigg, "Top-down fabricated silicon nanowires under tensile elastic strain up to $4.5 \%$," Nat Commun, vol. 3 , p. 1096, Oct. 2012. doi: 10.1038/ncomms 2102

[33] N. Waldron, C. Merckling, L. Teugels, P. Ong, S. Ibrahim, F. Sebaai, A. Pourghaderi, K. Barla, N. Collaert, and A.-Y. Thean, "InGaAs Gate-All-Around Nanowire Devices on 300mm Si Substrates," Electron Device Letters, IEEE, vol. 35, no. 11, pp. 1097-1099, Nov 2014. doi: 10.1109/LED.2014.2359579

[34] T. Bahder, "Eight-band k.p model of strained zinc-blende crystals," Physical Review B, vol. 41, pp. 11992-12001, June 1990. doi: 10.1103/PhysRevB.41.11992

[35] R. Neffati, I. Saidi, and K. Boujdaria, "Full-zone k.p model for the electronic structure of unstrained GaAs1xPx and strained AlxIn1xAs alloys," Journal of Applied Physics, vol. 112, no. 5, p. 053716, Sept. 2012. doi: $10.1063 / 1.4751353$

[36] I. Vurgaftman, J. R. Meyer, and L. R. Ram-Mohan, "Band parameters for III-V compound semiconductors and their alloys," Journal of Applied Physics, vol. 89, no. 11, p. 5815, June 2001. doi: 10.1063/1.1368156 